\title{
Participantes heterogéneos en MOOC y sus necesidades de aprendizaje adaptativo
}

\section{Heterogeneous Users in MOOC and their Adaptive Learning Needs}

\author{
Dolores Lerís ${ }^{1}$, María Luisa Sein-Echaluce 1, Miguel Hernández 2, Ángel Fidalgo-Blanco 3

\begin{abstract}
Universidad de Zaragoza. Zaragoza, España. \{dleris, mlsein\}@unizar.es
Universidad Católica de Valencia. Valencia, España. miguel.hernandez@ucv.es
\end{abstract} \\ Universidad Politécnica de Madrid. Madrid, España. angel.fidalgo@upm.es
}

\section{Resumen}

En numerosos estudios se señalan la masificación y la diversidad de perfiles de los participantes en los cursos masivos abiertos online (MO0C) como las principales causas de su baja tasa de finalización. Por otra parte, las metodologías de personalización del aprendizaje, junto a las tecnologías de la información, que permiten realizar técnicas de adaptatividad, figuran en informes internacionales como una forma eficaz de mejorar el aprendizaje. En este trabajo se explora la percepción de los participantes en MOOC respecto a sus necesidades de adaptatividad en este tipo de cursos, así como su relación con distintos aspectos de los participantes, como sus perfiles (género, edad, ubicación geográfica y nivel académico), su experiencia previa o conocimiento sobre el tema del M00C y su motivación para iniciar el M00C. Se realiza el estudio a través de una encuesta cumplimentada por los participantes en el Campus MO0C de Innovación Educativa. Se concluye que la edad o género de los participantes no influye significativamente en su necesidad de técnicas adaptativas en un MOOC. Sin embargo, residir en un país de América Latina, tener una profesión de gestión o inscribirse en un M00C con una motivación concreta son algunos de los factores que influyen en un mayor deseo de técnicas de adaptatividad en un MOOC. Los resultados obtenidos contribuirán a mejorar los diseños adaptativos de los MOOC y son fácilmente transferibles a cualquier curso de formación en línea, en formación semipresencial o virtual.

\section{Palabras Clave}

Aprendizaje adaptativo; MOOC; aprendizaje a distancia; educación abierta.

\section{Abstract}

Many research works point out the overcrowding and the heterogeneity of participant's profiles in Massive Open Online Courses (MOOC) as the main causes of their low completion rate. On the other hand, the methodologies of personalization of the learning, along next to the technologies of the information, that allows to realize techniques of adaptativity, appear in international reports as an effective way to improve the learning. This paper explores the participants' perception of their adaptive needs in this type of course, as well as their relationship with different aspects of the participants, such as: profiles (gender, age, geographical location and academic level), previous experience and knowledge about the topic of the MOOC and motivation to enroll the MOOC. The study is carried out through a survey completed by the participants in the MOOC Campus of Educational Innovation. We conclude that the age or gender of the participants does not significantly influence their need for adaptive techniques in a MOOC. However, living in a Latin American country, working as a manager or enrolling in a MOOC with a specific motivation, are some of the factors that influence in the desire for adaptive techniques in a MOOC. The obtained results will contribute to improve the adaptive designs of the MOOC and will be easily transferable to any online training course, in blended or virtual learning.

\section{Keywords}

Adaptive learning; MOOC; distance learning; open education. 


\section{Introducción}

El movimiento OpenCourseWare (OCW) fue iniciado por el Massachusetts Institute of Technology (MIT), a principios de 2001, en respuesta al creciente progreso de la informática y las comunicaciones como facilitadores del intercambio de información (Abelson, 2008). Entre los objetivos de OCW se encontraban el proporcionar acceso gratuito y abierto a todos los materiales de los cursos del MIT y la creación de un modelo eficiente, basado en estándares, que aporte valor al profesorado del MIT y que otras universidades puedan imitar (OCW, 2002).

Los cursos masivos abiertos en línea (Massive Open Online Courses: MOOC) surgieron como una evolución del movimiento OCW (Fidalgo-Blanco, Sein-Echaluce Lacleta, Borrás Gené \& García-Peñalvo, 2014). El primer curso, denominado MOOC en la literatura, fue creado por George Siemens y Stephen Downes, como un curso en línea introductorio, para promocionar su máster, y que contó con más de 2.200 inscritos (Downes, 2008). Este curso combinaba el uso de la plataforma de e-learning Moodle (Fini, 2009), con otras herramientas de comunicación y colaboración entre los estudiantes, como facebook, wikis, blogs, etc.

Desde la creación del primer MOOC, se identifican dos tipos de MOOC, los cMOOC y los XMOOC. Los cMOOCs son los primeros que aparecieron (Downes, 2008) y se basan en enfoques educativos conectivistas. Los XMOOCs se parecen a los cursos en línea tradicionales, dando la máxima prioridad a los materiales creados por el profesorado del curso (Fidalgo-Blanco, Sein-Echaluce \& GarcíaPeñalvo, 2015; 2016). Plataformas de e-learning para MOOC como edX (MIT), Coursera (Stanford) (Stone, 2016) y MiriadaX (MiriadaX, 2016), la más utilizada en español, producen cursos tipo xMOOC.

Los MOOC permiten el acceso a la educación superior de todas las personas para que, de ese modo, puedan participar activamente en su propio aprendizaje. Por tanto, estos cursos masivos abiertos en línea favorecen la inclusión social de las personas en la educación, difunden el conocimiento formal e informal a un gran público y facilitan la innovación de los docentes en sus acciones pedagógicas (Teixeira, et al., 2015). Así mismo, algunas de las modalidades de MOOC, propician la creación de comunidades de aprendizaje en torno a las temáticas de los mismos (Cruz-Benito, et al., 2015; Cruz Benito, et al., 2017).

Por otro lado, la masificación y la heterogeneidad de los perfiles de los participantes (idioma, intereses, ubicación geográfica, formación académica, actividad profesional, experiencia previa, motivación, etc.) son las dos características principales de los MOOC, en comparación con los cursos en línea tradicionales. Estas características enriquecen el contexto de formación y cooperación, pero dificultan su gestión. 
Una de las principales consecuencias de estas características de los MOOC es la alta tasa de abandono en este tipo de cursos. La tasa de finalización es una variable utilizada habitualmente para medir la calidad de un MOOC (Brahimi \& Sarirete, 2015) y la mayoría de ellos muestran un promedio de finalización de 6,5\%. Pero si consideramos solamente a los estudiantes activos; es decir, aquellos que han visto al menos un video, o han realizado al menos un intento de cuestionario, el promedio asciende al 9,8\% (Jordan, 2014).

Todo lo anterior hace necesaria la inclusión de capacidades adaptativas en los MOOC, así como en el aprendizaje en línea o en el aprendizaje semipresencial, que permitan personalizar el aprendizaje para disminuir la tasa de abandono provocada, en muchos casos, por la masificación y la heterogeneidad de esos cursos (Lerís \& Sein-Echaluce, 2011; Lerís López, Vea Muniesa \& Velamazán Gimeno, 2015).

La personalización del aprendizaje se señala como uno de los principales desafíos a los que se enfrentan las universidades y como uno de los desafíos más difíciles de cumplir (NMC Horizon Report, 2015). Las tecnologías para el aprendizaje adaptativo ocupan el primer lugar entre las 10 tecnologías estratégicas que impactan en la Educación Superior en 2016 (Gartner, 2016).

Con respecto a la tecnología, los sistemas hipermedia adaptativos representaron el fin de las estructuras estáticas a finales del siglo pasado, empezando por la adaptación de textos a los usuarios (Brusilovsky, 1996) y posteriormente adaptando los diseños de aprendizaje y los contenidos (Berlanga \& García-Peñalvo, 2005; 2008, Carro, 2001).

Otros trabajos recientes relacionados con el aprendizaje adaptativo para MOOC se centran en adaptar los contenidos a los conocimientos previos o incluso dependiendo del dispositivo móvil utilizado para acceder al curso. El trabajo de Lerís, Sein-Echaluce, Hernández y Bueno (2017) incluye estos trabajos entre otros dentro del estado de arte sobre esta temática. Este trabajo demuestra que es consistente un constructo de adaptatividad creado para identificar los indicadores de personalización más aceptables por los participantes en un MOOC.

Este trabajo completa el estudio mencionado y propone un análisis estadístico para determinar si la condición de adaptatividad es una preferencia de los usuarios de MOOC, dependiendo de algunas de sus características personales específicas. Las variables a estudiar son: factores demográficos (edad, género, ubicación geográfica y nivel de educación), conocimiento previo sobre la temática del MOOC y motivación para inscribirse en MOOC. El objetivo de este estudio es encontrar la relación de cada uno de estos factores con la importancia atribuida por los participantes a las acciones de aprendizaje de adaptatividad que podrían ser incluidas en un MOOC.

La siguiente sección presenta el contexto de la investigación, con las preguntas de investigación y las características de la muestra escogida. La sección de resultados contiene las respuestas a las 
preguntas de investigación y el trabajo finaliza con las conclusiones de este estudio.

\section{Metodología}

\subsection{Contexto}

El marco de referencia es la plataforma i-MOOC (http://gridlab.upm.es/imooc/), desarrollada como colaboración de la Universidad Politécnica de Madrid y la Universidad de Zaragoza en 2013, en la plataforma de e-learning de código abierto Moodle (Moodle, 2016) y al que se unió la Universidad de Salamanca en 2015.

En esta plataforma se implementó el "Campus MOOC de Innovación Educativa" (en adelante CMIE) en 2015-16 (Sein-Echaluce, Fidalgo-Blanco, García-Peñalvo \& Conde, 2016). Este Campus está compuesto por cuatro aMOOC (adaptive MOOC):

- aM00C: Fundamentos prácticos de la Innovación Educativa.

- aM00C: Flip Teaching.

- aMOOC: Comunidades de aprendizaje.

- aMOOC: Desarrollo de la competencia de trabajo en equipo.

Los cuatro aMOOC incluyen facilitadores adaptativos (proporcionados por la plataforma utilizada) en aspectos logísticos, metodológicos y tecnológicos, tales como: restricciones de acceso, condiciones de finalización, grupos y agrupaciones, así como varios plugins externos, como el que permite elegir el grupo, por ejemplo. De este modo, el aprendizaje se personaliza para cada participante proporcionando contenidos y actividades adaptados a diferentes intereses y características.

Se pidió a los participantes en los aMOOC que rellenaran una encuesta cuyos ítems muestran el constructo propuesto por Lerís et al. (2017) y descrito en la siguiente subsección.

\subsection{Preguntas de investigación}

El constructo definido previamente, y denominado baMOOC, corresponde a la percepción de los participantes en los aMOOC mostraron sobre la importancia del uso de la adaptatividad en MOOC. Este constructo se genera como la suma de las respuestas a 6 preguntas (Likert escala de cuatro niveles: "Nada de acuerdo", "Poco de acuerdo", "Bastante de acuerdo" y "Totalmente de 
acuerdo" puntuación de 1 a 4, respectivamente), por lo que toma valores entre 6 y 24 . En el estudio se han realizado diferentes comparaciones para encontrar una relación entre algunas características específicas de los participantes y el constructo baMOOC.

El constructo baM00C está compuesto por los siguientes ítems (Lerís et al., 2017):

- Ítem 1. Prefiero que me propongan diferentes actividades dependiendo de mi elección o de mis resultados de evaluación.

- Ítem 2. Prefiero poder acceder a contenidos / actividades siguiendo mi ritmo de trabajo, no un calendario predeterminado para acceder a los contenidos / actividades.

- Ítem 3. Prefiero poder elegir entre diferentes niveles de dificultad en los contenidos / actividades dependiendo de diferentes objetivos de aprendizaje.

- I Ítem 4. Prefiero que se creen grupos de interés (misma área, mismo nivel de experiencia, etc.) para debatir en foros específicos.

- I Ítem 5. Prefiero poder elegir entre diferentes métodos de evaluación (autoevaluación, revisión por pares, etc.).

- Ítem 6. Prefiero que la revisión por pares también esté organizada por grupos de interés (misma área, mismo nivel de experiencia, etc.).

Se consideran tres factores como variables a comparar con el constructo baM00C, aunque algunos de ellos tienen varios subfactores:

1. Factores demográficos. Entre los factores demográficos considerados se encuentran el género, la edad, la ubicación geográfica y el nivel de formación académica previa.

2. Experiencia previa en la temática del MOOC. Se evalúa si el hecho de tener conocimiento del contenido en el que se basa el MOOC puede producir que cambie la valoración de los participantes sobre la adaptatividad.

3. Motivación para inscribirse en el MOOC. Se realiza un estudio estadístico para verificar si las razones aducidas para inscribirse en el MOOC pueden estar relacionadas con la percepción de la valoración que el participante tiene sobre la adaptatividad.

Con todo lo anterior se han establecido las siguientes hipótesis de investigación en relación a los tres factores mencionados y la adaptatividad en un MOOC a través del constructo baMOOC:

- RQ1. ¿Factores demográficos, como el género, la edad, la ubicación geográfica y la formación 
académica previa, influyen en el valor que los participantes en un MOOC dan a las acciones de adaptatividad?

- RQ2. ¿La experiencia o los conocimientos previos en la temática del MOOC influyen en el valor que los participantes en el MOOC dan a las acciones de adaptatividad?

- RQ3. ¿La motivación para inscribirse en un MOOC influye en la percepción del valor que los participantes en un MOOC dan a las acciones de adaptatividad?

\subsection{Características de la muestra}

El CMIE fue diseñado de forma que la encuesta inicial era el único recurso disponible para los participantes antes de tener acceso a cualquier aMOOC. Por lo tanto, todos los inscritos en el campus debían responder esa encuesta inicial, como condición previa para ver y acceder a otras actividades. En esa situación, la muestra se compone de 475 participantes que accedieron a CMIE entre noviembre de 2015 y febrero de 2016.

La encuesta inicial, diseñada por los autores, contiene las preguntas pertinentes para recopilar los datos necesarios y responder a las preguntas de investigación.

\section{Resultados}

En este apartado exponemos los resultados obtenidos al analizar la posible influencia de ciertas características personales en el valor concedido por los participantes a la variable baMOOc, y, en particular, a los ítems que la conforman.

Como ya se indicó en Lerís et al. (2017), ha de rechazarse la normalidad de la variable baMO0C, ya que tanto el test de Shapiro-Francia $(\mathrm{W}=0.92352$, $\mathrm{p}$-value $=4.145 \mathrm{e}-13)$ como el de KolmogorovSmirnov (Kolmogorov distance $=0.11074, \mathrm{p}$-value $=1.744 \mathrm{e}-05)$, aplicados a la muestra, dan lugar a p-valores inferiores a 0.05. En consecuencia, el análisis estadístico que se ha realizado utiliza técnicas estadísticas del llamado análisis no paramétrico.

Así, en el caso de las variables dicotómicas, aquellas que definen dos grupos o muestras, como el género, se ha utilizado de forma sistemática la prueba de Wilcoxon (o Wilcoxon-Mann-Whitney), de dos muestras independientes, para testar la igualdad de la distribución (hipótesis nula) de la variable baM00C y la de las variables que la componen, para ambos grupos de la muestra. En los casos en que los resultados de la prueba de Wilcoxon han llevado a rechazar la hipótesis nula, se ha procedido a analizar el tamaño de la diferencia mediante la delta de Cliff. En otros casos, y si el análisis descriptivo lo ha sugerido, se ha estudiado si existe diferencia en el grado de variabilidad de las dos distribuciones 
mediante la prueba de reacciones extremas de Moses.

En cuanto a las variables politómicas, aquellas en las que el número de categorías es superior a dos, como por ejemplo el intervalo de edad en el que se sitúa el participante, se ha utilizado la prueba de Kruskal-Wallis (extensión de la de Wilcoxon-Mann-Whitney a varias muestras independientes) para testar la hipótesis nula de que todos los grupos proceden de poblaciones idénticas.

El análisis estadístico de los datos se ha realizado con R versión 3.2.2 (R Core Team, 2015) y los siguientes paquetes R: 'orddom' (Rogmann, 2013) para calcular el parámetro Delta de Cliffy 'DescTools' (Signorell, 2016) para obtener la prueba de reacciones extremas de Moses.

\subsection{Influencia de factores demográficos en la valoración de la adaptatividad (RQ1)}

Las cuatro características demográficas consideradas son: género, edad, ubicación geográfica y nivel educativo. Para cada uno de ellas se analiza si el constructo baMOOC y las variables que lo forman, son estadísticamente independientes de cada uno de esos factores.

\subsubsection{Género}

El factor género divide la muestra $(n=475)$ en dos grupos: 222 hombres y 253 mujeres.

La media y la desviación estándar de la variable baMOOC (que toma valores de 6 a 24) es muy similar entre ambos grupos (véase la Tabla 1). Por lo tanto, parece que la tendencia central y la variabilidad de ambos conjuntos de datos son similares. No obstante, los diferentes valores del coeficiente de asimetría y, especialmente, de la curtosis, mostrados en la Tabla 1, y los histogramas de la Figura 1, parecen revelar que estos conjuntos de datos tienden a tener diferentes colas o valores atípicos.

\begin{tabular}{|c|c|c|c|c|c|}
\hline Género & Media & Desviación estándar & Coeficiente de asimetría & Curtosis & $\mathbf{n}$ \\
\hline Hombre & 20.41 & 2.80 & -0.35 & -0.52 & 222 \\
\hline Mujer & 20.23 & 2.99 & -0.76 & 1.55 & 253 \\
\hline
\end{tabular}

Tabla 1. Medidas descriptivas de baM00C por género

En primer lugar, hemos testado la hipótesis nula de que las dos muestras, la de hombres y la de mujeres, se extraen de distribuciones con igual rango medio. Las medianas de los dos grupos son 20,5 para hombres y 20,0 para mujeres, y el estadístico de Wilcoxon es $\mathrm{W}=28800$ con un valor de $\mathrm{p}$ $=0,6276$. Por lo tanto, se acepta que el desplazamiento de la mediana es igual a cero, es decir, que la distribución de variable baMOOC es significativamente igual en ambos géneros.

En segundo lugar, debido a que en el grupo de hombres aparece con más frecuencia una valoración menor de la variable baMOOC, véase el aspecto bimodal del histograma de este grupo, hemos utilizado 
la prueba de reacciones extremas de Moses, para testar la igualdad entre los dos grupos (masculino y femenino) en los extremos de las puntuaciones (y en ambas direcciones). El valor del estadístico de Moses es S = 398 y el p-valor es 0.0005613: por lo tanto, la hipótesis nula es rechazada, es decir, los valores extremos son más probables en los hombres que en las mujeres.

Por último, en relación con el factor de género, cuando revisamos por separado los seis ítems de baM00C, todos los p-valores de la prueba de Wilcoxon-Mann-Whitney son mayores que 0.05. Por lo tanto, ha de aceptarse que las distribuciones de los seis ítems son iguales, independientemente del género.

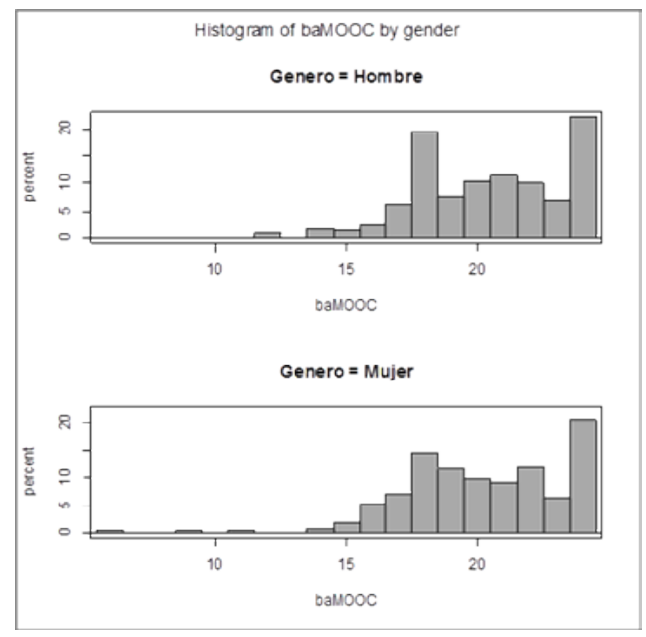

Figura 1. Histograma de las dos muestras de la variable baMOOC según el factor de género.Edad

\subsubsection{Edad}

De nuevo, la media y la desviación estándar son muy similares para los cinco intervalos de edad considerados, como se puede ver en la Tabla 2.

\begin{tabular}{|c|c|c|c|c|c|}
\hline Edad & Media & Desviación estándar & Coeficiente de asimetría & Curtosis & $\mathbf{n}$ \\
\hline Hasta 25 & 20.03 & 2.99 & -0.49 & 0.01 & 31 \\
\hline 26 a 35 & 20.48 & 3.10 & -1.249 & 3.87 & 89 \\
\hline 36 a 45 & 20.26 & 2.90 & -0.25 & -0.83 & 151 \\
\hline 46 a 55 & 20.54 & 2.77 & -0.59 & 0.57 & 157 \\
\hline Más de 55 & 19.60 & 2.85 & -0.44 & 0.52 & 47 \\
\hline
\end{tabular}

Tabla 2. Medidas descriptivas de baM00c por edad

Aplicando el test de Kruskal-Wallis para verificar la hipótesis de igualdad de distribuciones de la variable baMOOC a cinco intervalos de edad, los resultados son los siguientes: Kruskal-Wallis chi cuadrado = 4.9082 y p-valor $=0.2968$. Por lo tanto, se acepta que las cinco distribuciones son idénticas, es decir, la variable baMOOC es independiente del intervalo de edad considerado.

Aunque habiéndose demostrado que no hay diferencias significativas según el tramo de edad, es 
interesante ordenar los intervalos de edad en el orden creciente de sus medias en baM00C. La lista resultante se muestra en la Figura 2 y se puede ver la ubicación inferior, y más alejada, del grupo de participantes de más de 55 años.

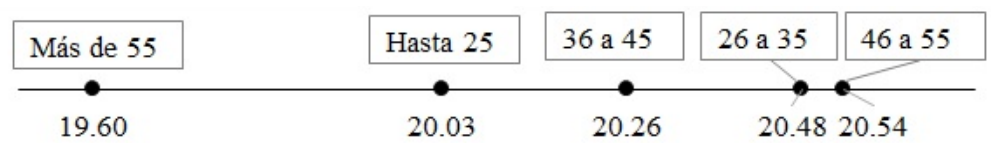

Figura 2. Medias de baM00C por edad

Por último, en relación con el factor edad, las medias de los seis ítems, que conforman el baM00C, proporcionan información sobre las diferencias en el comportamiento de cada grupo de edad. Así, en la Figura 3, se puede ver que las mayores diferencias se dan en los ítems 1, 2 y 4. De hecho, la prueba de Kruskal-Wallis aplicada a estos tres ítems proporciona p-valores alrededor de 0.16 que, si bien no son estadísticamente significativos, parecen revelar un comportamiento diferente especialmente en el ítem 4.

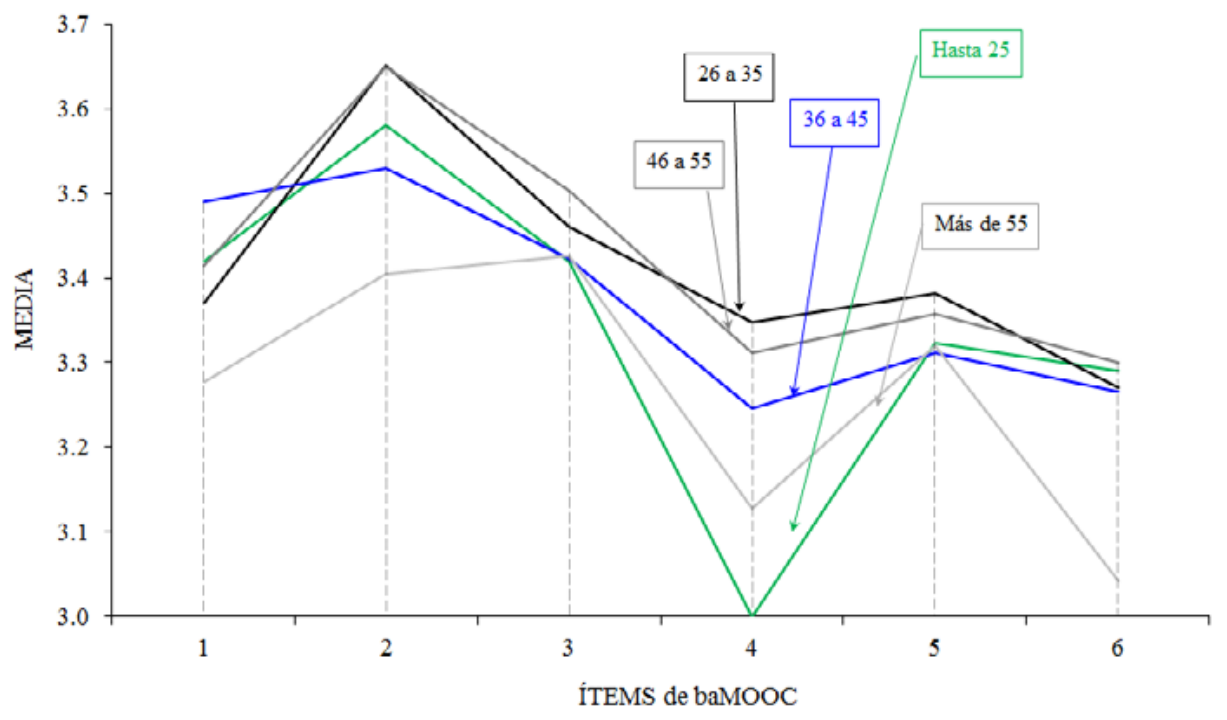

Figura 3. Medias de los seis ítems baMOOC por edad

\subsubsection{Localización geográfica}

En este estudio también se ha analizado la ubicación geográfica de los participantes como un factor que puede influir en la percepción del valor que tiene la adaptatividad en un MOOC. Los participantes en el CMIE respondieron a una pregunta de la encuesta en la que se les requería que indicaran el país en el que viven.

Los resultados se han agrupado en dos categorías: países de América ( $n=211,44.42 \%$ ) y países de Europa ( $n=264,55.58 \%$ ). Los participantes americanos son todos de América Latina, excepto uno de los Estados Unidos, y la mayoría de los participantes europeos residen en España ( $n=253,96 \%$ ).

En este caso, la ubicación geográfica de los participantes parece producir una mayor diferencia en las medias (véase la Tabla 3). Por otra parte, en la Figura 4, el diagrama de caja de la variable baMOOC para 
cada grupo de participantes, según su lugar de residencia, permite apreciar un ligero diferencia en la distribución de los residentes americanos, en su preferencia por los valores superiores de la variable, respecto a los europeos. La prueba de suma de rangos de Wilcoxon proporciona los siguientes valores: $W=31.230$ y $p$-valor $=0.02177$. Por lo tanto, rechazamos la hipótesis nula del test, es decir, aceptamos que hay una diferencia significativa entre las dos distribuciones. En otras palabras, la variable baMOOC se ve afectada por el factor de continente de residencia de los participantes.

\begin{tabular}{|c|c|c|c|c|c|}
\hline Edad & Media & Desviación estándar & Coeficiente de asimetría & Curtosis & $\mathbf{n}$ \\
\hline América & 20.58 & 3.08 & -0.80 & 0.39 & 211 \\
\hline Europa & 20.10 & 2.73 & -0.44 & 1.36 & 264 \\
\hline
\end{tabular}

Tabla 3. Medidas descriptivas de baM00c por localización geográfica

Para poder evaluar el efecto de este factor, utilizamos un estadístico del grupo de los denominados Common Language Effect Size. En este caso, la probabilidad de que los participantes americanos aprecien más la adaptatividad que los europeos, es estimada por la proporción del número de veces que un dato del grupo americano tiene un valor mayor que un dato del grupo europeo. En este caso, este valor es 0.4999 frente a la superioridad contraria (visión europea sobre americana) cuyo valor es 0.3786. Por lo tanto, el valor del parámetro Delta Cliff es 0.1213, que es la diferencia entre las probabilidades de superioridad antes mencionadas.

En resumen, existe una diferencia significativa entre la percepción de los participantes americanos y europeos sobre el valor de la adaptatividad básica en un MOOC. Esta diferencia se ha estimado en 0.1213 (la diferencia entre las probabilidades de superioridad en una dirección y en la inversa) y, aunque pequeña, debería tenerse en cuenta en el diseño de un MOOC.

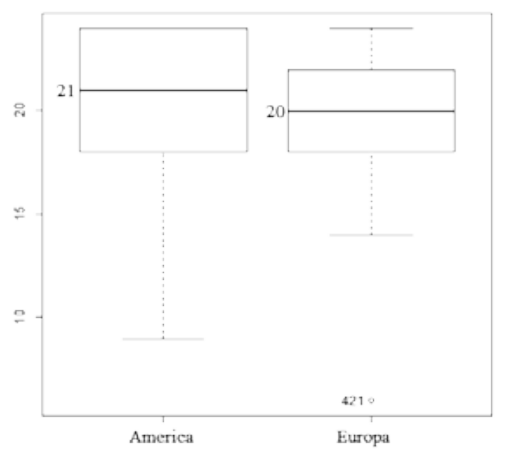

Figura 4. Diagrama de caja de la variable baM00C por localización geográfica

Cuando se analiza la independencia de cada uno de los seis ítems que conforman el constructo baMOOC, respecto al factor "continente" de residencia, los resultados de la prueba de Wilcoxon muestran que hay diferencias significativas en las distribuciones de los ítems 5 y 6 , los relacionados con la cooperación (Tabla 4). Para estos ítems, los tamaños de efecto, medidos por la Delta de Cliff son 0.1085 y 0.1636 , respectivamente. 


\begin{tabular}{|c|c|c|c|}
\hline Ítem & $\begin{array}{c}\text { Medianas } \\
\text { America-Europa }\end{array}$ & Estadístico de Wilcoxon & p-valor \\
\hline Ítem 5 & $4-3$ & $W=30.874$ & 0.02415 \\
\hline Ítem 6 & $3-3$ & $W=30.904$ & 0.0239 \\
\hline
\end{tabular}

Tabla 4. Resultados del test de Wilcoxon de los ítems 5 y 6 de baM00C por localización geográfica

\subsubsection{Localización geográfica}

El historial educativo de los participantes es el último factor demográfico que consideramos. La formulación de la correspondiente pregunta en la encuesta es: "Su nivel de educación es ... (con seis opciones)". Las 475 respuestas recogidas se desglosan de la siguiente manera: 4 (0.84\%) de Educación Primaria; 15 (3.16\%) de Educación Secundaria; 35 (7.37\%) de Formación Profesional; 212 (44.63\%) de Educación Superior - Graduados y 198 (41.68\%) de Educación Terciaria - Máster o Doctorado, y 11 $(2.32 \%)$ de otros.

Por lo tanto, la conclusión más importante es que más del 95\% de los participantes han completado alguna educación terciaria. Debido a ello, hemos restringido el análisis estadístico a este tipo de participantes, que hemos desagregado en dos grupos, uno para graduados universitarios y el otro para masters o doctorados.

Como se puede ver en la Figura 5, la distribución de la variable baMOOC es muy similar en ambos grupos. Por otra parte, la prueba de la suma de rangos de Wilcoxon ( $\mathrm{W}=20.144$ y p-valor $=0.4774$ ) permite aceptar la hipótesis nula de la igualdad de distribuciones.
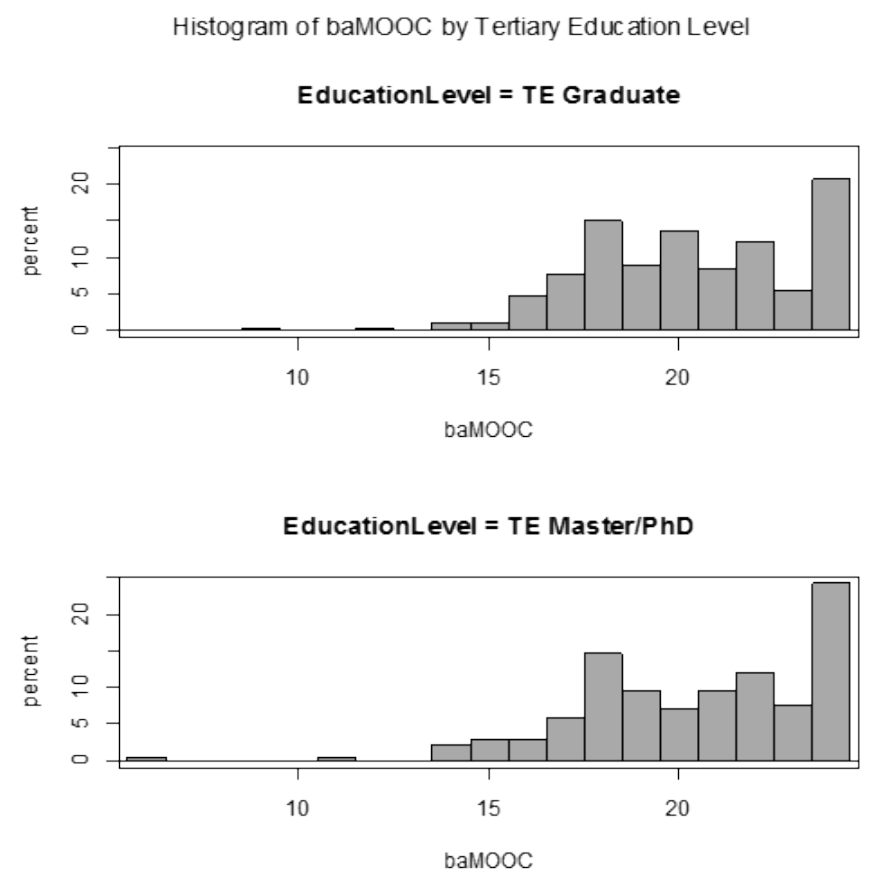

Figura 5. Histogramas de la variable baM00C por nivel educativo

Al comparar las distribuciones de cada uno de los seis ítems (cuya agregación conforma la variable baMOOC) según el nivel de educación terciaria, la prueba no paramétrica de Wilcoxon muestra que 
son significativamente iguales excepto para el ítem $4(W=23.305$ y p-valor $=0.03436)$. El tamaño del efecto medido por la Delta de Cliff para ese ítem 4 es 0.1104. Es decir, la diferencia entre la probabilidad de que un sujeto elegido aleatoriamente del grupo de master / doctorado otorgue mayor puntuación en el ítem 4, que un miembro, elegido al azar, del grupo de graduados (que es del 36\%) y la contraria (24.96\%) se estima en $11.04 \%$. Existe una probabilidad del 95\% de que el verdadero valor Delta de Cliff se encuentre entre 0.0081 y 0.2104 .

\subsection{Influencia de la experiencia previa en la temática, sobre la valoración de adaptatividad (RQ2)}

Como se ha mencionado anteriormente, la temática del CMIE fue la innovación educativa. Por lo tanto, se preguntó a los participantes sobre su experiencia previa en ese tema, del siguiente modo: ¿Tiene experiencia previa en innovación educativa? Las cuatro opciones de respuesta y sus frecuencias se muestran en la Tabla 5. Se resalta en negrita la opción C, porque presenta la máxima frecuencia de respuestas.

\begin{tabular}{|c|c|}
\hline Opciones & N \\
\hline A. No tengo experiencia previa en innovación educativa & 101 \\
\hline B. Solo he visto información en internet sobre innovación educativa & 146 \\
\hline C. He aplicado o estoy aplicando innovación educativa & 183 \\
\hline D. He ocupado o estoy ocupando un puesto de gestión relacionado con la innovación educativa & 45 \\
\hline
\end{tabular}

Tabla 5. Frecuencias de respuestas sobre experiencia previa en innovación educativa

Al analizar los datos de la variable baMOOC, se aprecia cierta diferencia entre los grupos A, B y C y el grupo D, como muestra la Figura 6. Esta diferencia aparente no es estadísticamente significativa a un nivel del 95\% (Kruskal-Wallis chi-cuadrado $=5.0893$, p-valor $=0.1654$ ), pero destaca el interés de los gestores en proveer adaptatividad a los usuarios de los MOOC. Además, todos los ítems que componen la variable baM00C siguen un patrón similar. De hecho, la gráfica de la Figura 7 muestra que las medias de todos los ítems del grupo D (Gestores) son mayores que en los otros grupos, aunque sus diferencias no son estadísticamente significativas. Solo observamos que las medias de los ítems 5 y 6 (relacionados con la cooperación) del grupo D parecen estar más cerca de los de los otros grupos.

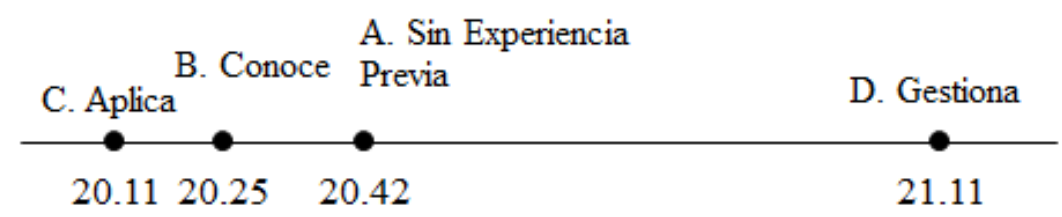




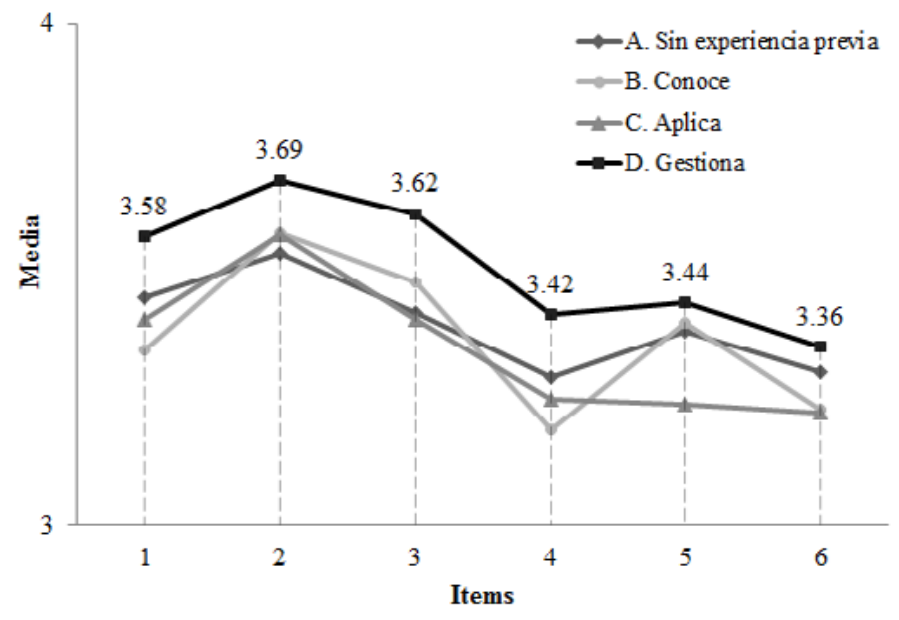

Figura 7. Medias de los ítems de respuesta sobre experiencia previa en innovación educativa

\subsection{Influencia de la motivación sobre la valoración de la adaptatividad (RQ3)}

El último factor a considerar es la motivación de los participantes para inscribirse en el CMIE. La encuesta realizada incluye una pregunta sobre ese tema. En concreto, la formulación de la pregunta es "Marque la razón o razones por las que se ha inscrito en este Campus de Innovación Educativa". Las seis opciones de respuestas y sus frecuencias se incluyen en la Tabla 6. Hay que tener en cuenta que cada participante puede marcar tantas opciones como estime conveniente, por lo tanto, la suma de las frecuencias es mayor que el número de participantes.

A continuación, en la Figura 8 se muestran conjuntamente las siguientes dos líneas: la línea de trazo negro corresponde a la proporción de participantes que marcaron cada opción de respuesta y la línea gris refleja la proporción de los que seleccionaron solo una de las opciones. Como era previsible, la opción A alcanza las mayores proporciones en ambos casos, pues en ella se expresa una razón muy general para inscribirse en un curso de formación. Después de ella, podemos observar que las opciones $\mathrm{C}, \mathrm{D}$ y $\mathrm{E}$, que a nuestro juicio declaran objetivos claros, alcanzan mayores frecuencias de respuesta que las otras dos opciones $B$ y F, en las que se expresan objetivos más vagos.

\begin{tabular}{|c|c|}
\hline Opciones & N \\
\hline A. Para aumentar mi conocimiento en términos generales & 253 \\
\hline B. Para aprender sobre innovación educativa, aunque no la aplique ahora & 63 \\
\hline C. Para empezar a aplicar la innovación educativa & 169 \\
\hline D. Para tener una nueva visión sobre la innovación educativa & 204 \\
\hline E. Para investigar sobre innovación educativa & 217 \\
\hline F. Para conocer la estructura del curso y/o acceder a sus materiales & 91 \\
\hline
\end{tabular}

Tabla 6. Frecuencias de los ítems de respuesta sobre motivación para inscribirse en el campus iMOOC 
Consideremos, ahora, cada tipo de motivación como un factor que divide la muestra en dos grupos (los participantes que lo han marcado y los que no lo han hecho) y examinemos si las distribuciones de la variable baMOOC y de sus ítems son iguales en estos dos grupos. Los resultados se exponen a continuación.

En primer lugar, los dos grupos de la opción A (los que la han marcado y los que no) no difieren en su percepción del valor global de la adaptatividad en un MOOC, pero hay diferencias significativas en los ítems 3 y 4.

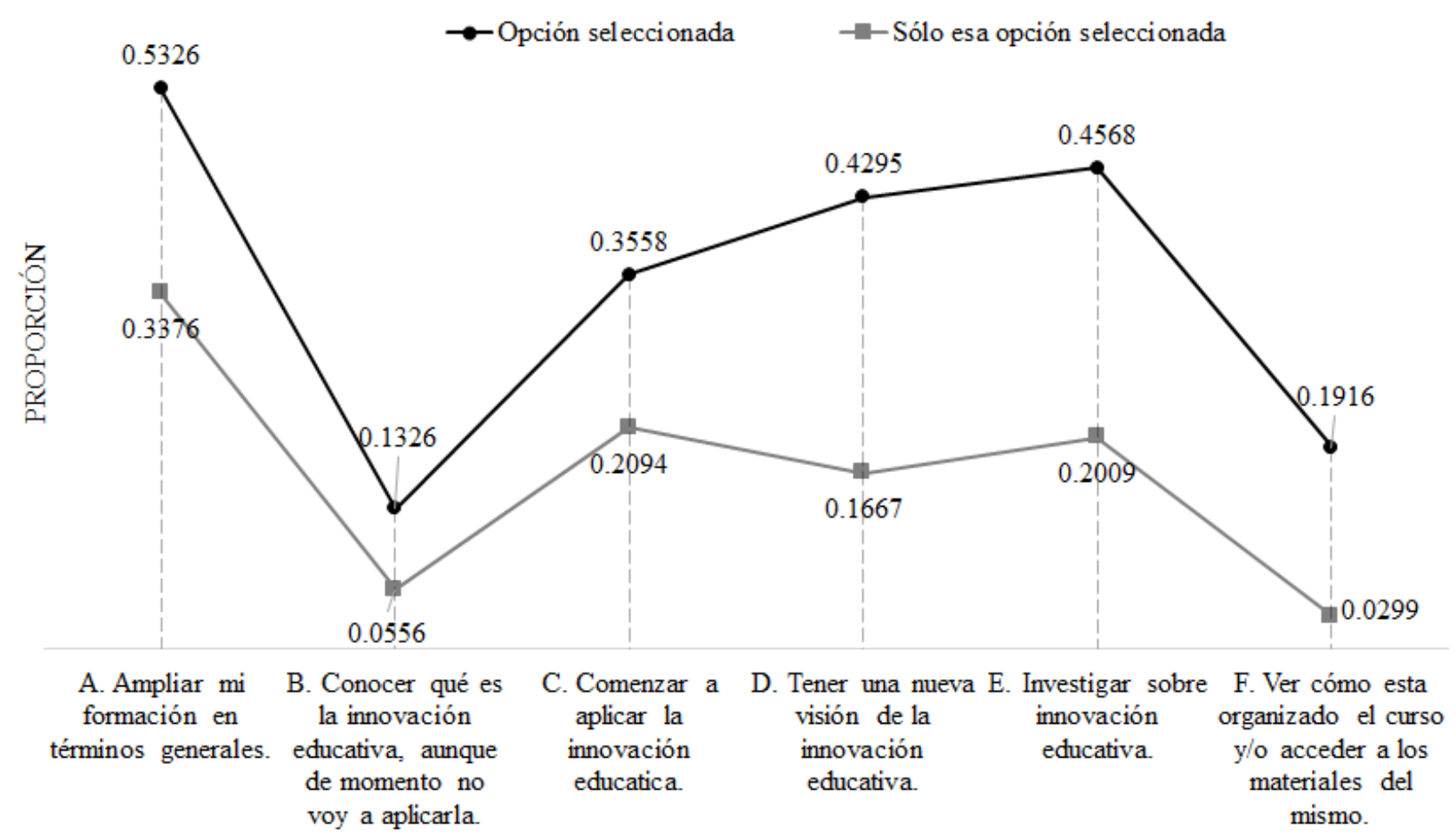

Figura 8. Proporción en la que es seleccionada cada opción de motivación

En segundo lugar, cuando analizamos las opciones C, D y E, la prueba de Wilcoxon permite afirmar que solo hay diferencias entre el grupo que ha marcado la motivación E y el que no lo ha marcado. En este caso, las medianas son 21 y 20 para los que sí la marcan y para los que no lo marcan, respectivamente; el estadístico de Wilcoxon es 24.901 y el p-valor es 0.03062. Por lo tanto, los participantes motivados por realizar investigación prefieren, de forma significativa, el diseño de adaptatividad en un MOOC.

Por último, con respecto a las opciones B y F, nuevamente la prueba de Wilcoxon muestra que no hay diferencias en las distribuciones de la variable baMOOC para cada grupo considerado (los que la marcan frente a los que no lo hacen). Sin embargo, la distribución de los ítems, relacionados con la capacidad de autorregulación, es significativamente diferente. En particular, los participantes que marcaron la motivación B valoran menos el ítem 2 que el grupo que no la marcó (mediana en ambos casos $=4, W=15.112, p$-valor $=0.0118)$. Por el contrario, los ítems 1 y 3 son más valorados por aquellos que marcaron la opción F; en efecto, los datos obtenidos son los siguientes: 
- Ítem 1: mediana del grupo que sí marcó la opción $\mathrm{F}=4$, mediana del grupo que no marcó la opción $F=3, W=15036$, p-valor $=0.01965$.

- Ítem 2: mediana del grupo que sí marcó la opción F = 4, mediana del grupo que no marcó la opción $F=3.5, W=15426$, p-valor = 0,04996.

\section{Conclusiones}

En este artículo se presentan los resultados de una investigación exploratoria sobre la influencia de diferentes factores en el valor del concepto de adaptatividad básica de un MOOC.

Los datos, utilizados para alcanzar los resultados presentados en este estudio, provienen de las respuestas a una encuesta implementada en el campus CMIE, formado por cuatro MOOC y apoyado por la plataforma Moodle. El idioma (español) y la temática del campus CMIE (la innovación educativa) limitan el alcance de los resultados obtenidos. Sin embargo, pueden ser considerados como resultados de un estudio preliminar sobre la percepción de los estudiantes en relación a la importancia de diseñar MOOC adaptativos.

El perfil del participante promedio en CMIE es el de una persona de mediana edad, residente en un país de habla hispana y graduado universitario. Los resultados del análisis de la influencia de cuatro factores demográficos (género, edad, ubicación geográfica y nivel educativo), en la variable baMOOC y cada uno de sus ítems, nos llevan a las conclusiones que señalamos a continuación.

La percepción de los participantes sobre el diseño básico de adaptatividad en un MOOC, difiere dependiendo de su continente de residencia. En concreto, los residentes en América Latina indican una mayor preferencia por la adaptatividad en un MOOC que los residentes en Europa. Además, existe una diferencia significativa, en el mismo sentido, en los dos indicadores relacionados con la evaluación (ítems 5 y 6).

Respecto al nivel educativo de los participantes, se observa que la organización de debates en foros, de acuerdo al área de interés / fondo académico / nivel de experiencia, es decir, el ítem 4, es más valioso para el grupo que posee nivel de máster/doctorado que para los graduados universitarios.

Sin embargo, ni el género ni el tramo de edad son factores que afecten significativamente la percepción de los participantes sobre el valor de la adaptatividad (definida en baMOOC) en el diseño de un MOOC.

Por otro lado, con respecto a la influencia de la experiencia previa, en la temática del campus CMIE, sobre el valor que dan los participantes a la adaptatividad, los resultados indican que no hay diferencia 
significativa entre los cuatro niveles de experiencia considerados en esta investigación. Sin embargo, cabe señalar que el grupo de gestores de la innovación educativa (nivel D) da valor a la adaptatividad por encima de cada uno de los otros tres grupos.

La motivación para realizar un curso de formación es un tema muy importante, especialmente en Ios MOOC, por lo que se ha analizado si algunas de las seis razones, consideradas para el ingreso en el CMIE, han influido en la percepción del valor de la adaptatividad en un MOOC. Se observa que solo los participantes interesados en la investigación sobre la temática del MOOC prefieren un diseño adaptativo del curso, por lo que parece que tener un objetivo tan claro en mente es un factor decisivo en la valoración de la adaptatividad en los cursos. Sin embargo, en el caso de las dos opciones de motivación B y F (véase Figura 8), que se refieren a objetivos más difusos, los indicadores relacionados con la autorregulación tienen diferentes distribuciones. Esto sugiere que la capacidad de organizar el propio aprendizaje en un MOOC está relacionada con objetivos claros para inscribirse.

Finalmente, para encontrar el mejor diseño de MOOC que mejore las tasas de finalización de los mismos, la propuesta de este trabajo es realizar MOOC con un diseño de adaptatividad. La percepción de los participantes acerca de la adaptatividad básica, mostrada en este trabajo, aporta ideas a tener en cuenta por los diseñadores de MOOC. En trabajos futuros se incluirán cambios en el diseño adaptativo de los aMOOC como, por ejemplo, diferentes actividades dependiendo del nivel académico o diferentes niveles de adaptatividad dependiendo de la ubicación geográfica. El objetivo es obtener mejores experiencias de aprendizaje para obtener mejores tasas de finalización en los MOOC y, como consecuencia, transferir esas experiencias a la docencia semipresencial o virtual, que poseen condiciones menos extremas que los MOOC.

\section{Agradecimientos}

Los autores de este trabajo agradecen el apoyo del Gobierno de Aragón, el Fondo Social Europeo y el servicio GATE de la Universidad Politécnica de Madrid, así como a sus grupos de investigación GIDTIC, http://gidtic.com y LITI, http://www.liti.es.

\section{Referencias}

Abelson, H. (2008). The Creation of OpenCourseWare at MIT. Journal of Science Education and Technology. 17(2), 164-174. doi:https://doi.org/10.1007/s10956-007-9060-8 
Berlanga, A. J., \& García-Peñalvo, F. J. (2005). Learning Technology Specifications: Semantic Objects for Adaptive Learning Environments. International Journal of Learning Technology. 1(4), 458-472. doi:https://doi.org/10.1504/IJLT.2005.007155

Berlanga, A. J., \& García-Peñalvo, F. J. (2008). Learning Design in Adaptive Educational Hypermedia Systems. Journal of Universal Computer Science. 14(22), 3627-3647. doi:https://doi.org/10.3217/jucs014-22-3627

Brahimi, T., \& Sarirete, A. (2015). Learning outside the classroom through MOOCs. Computers in Human Behavior, 51, 604-609. doi:http://dx.doi.org/10.1016/j.chb.2015.03.013

Brusilovsky, P. (1996). Methods and techniques of adaptive hypermedia. User Modeling and UserAdapted Interaction. 6(2), 87-129. doi:http://dx.doi.org/10.1007/BF00143964

Carro, R. M. (2001). Un mecanismo basado en tareas y reglas para la creación de sistemas hipermedia adaptativos: aplicación a la educación a través de Internet. PhD thesis.

Cruz-Benito, J., Borrás-Gené, O., García-Peñalvo, F. J., Fidalgo-Blanco, A., \& Therón, R. (2015). Detection of non-formal and informal learning in Learning Communities supported by social networks in the context of a cooperative MOOC. In 2015 International Symposium on Computers in Education (SIIE) (pp.195-200). USA: IEEE. doi:https://doi.org/10.1109/SIIE.2015.7451675

Cruz-Benito, J., Borrás-Gené, O., García-Peñalvo, F. J., Fidalgo-Blanco, A., \& Therón, R. (2017). Learning communities in social networks and their relationship with the MOOCs. IEEE Revista Iberoamericana de Tecnologias del Aprendizaje. In press.

Downes, S. (2008). Places to Go: Connectivism \& Connective Knowledge. Innovate: Journal of Online Education, 5(1), Article 6. Recuperado de http://nsuworks.nova.edu/innovate/vol5/iss 1/6

Fidalgo-Blanco, Á., Sein-Echaluce Lacleta, M. L., Borrás Gené, O. \& García-Peñalvo, F. J. (2014). Educación en abierto: Integración de un MOOC con una asignatura académica. Education in the Knowledge Society (EKS), 15(3), 233-255.

Fidalgo-Blanco, Á., Sein-Echaluce Lacleta, M. L., \& García-Peñalvo, F. J. (2015). Methodological Approach and Technological Framework to break the current limitations of MOOC model. Journal of Universal Computer Science, 21(5), 712-734. doi:https://doi.org/10.3217/jucs-021-05-0712

Fidalgo-Blanco, A., Sein-Echaluce, M. L., \& García-Peñalvo, F. J. (2016). From massive access to cooperation: lessons learned and proven results of a hybrid xMOOC/CMOOC pedagogical approach to MOOCs. International Journal of Educational Technology in Higher Education, 13(1), 1-13. doi:https:// doi.org/10.1186/s41239-016-0024-z 
Fini, A. (2009). The Technological Dimension of a Massive Open Online Course: The Case of the CCK08 Course Tools. The International Review of Research in Open and Distributed Learning, 10(5). doi:https:// doi.org/10.19173/irrodl.v10i5.643

Gartner. (2016). Top 10 Strategic Technologies Impacting Higher Education in 2016. Recuperado de https://www.gartner.com/doc/3186323?ref=SiteSearch\&sthkw=education top\&fnl=search\&srcld=1-3478922254

Jordan, K. (2014). Initial trends in enrolment and completion of massive open online courses. The International Review of Research in Open and Distributed Learning, 15(1), 133-160. doi: https://doi. org/10.19173/irrodl.v15i1.1651

Lerís, D., \& Sein-Echaluce, M. L. (2011). La personalización del aprendizaje: un objetivo del paradigma educativo centrado en el aprendizaje. Arbor, 187, Extra_3, 123-134. doi:https://doi.org/10.3989/ arbor.2011.Extra-3n3135

Lerís, D., Sein-Echaluce, M. L., Hernández, M., \& Bueno, C. (2017). Validation of indicators for implementing an adaptive platform for MOOCs. Computers in Human Behavior. In press. http://dx. doi. org/10.1016/j.chb.2016.07.054

Lerís López, D., Vea Muniesa, F., \& Velamazán Gimeno, Á. (2015). Aprendizaje adaptativo en Moodle: tres casos prácticos. Education in the Knowledge Society (EKS), 16(4), 138-157. doi:https://doi. org/10.14201/eks201516138157

MiriadaX. (2016). https://miriadax.net

MIT-OCW. (2002). Measuring Long-Term Success Evaluation of MIT OCW Depends on Articulation of Clear Goals. Recuperado de http://web.mit.edu/fnl/vol/155/han.htm

Moodle. (2016). https://moodle.org

NMC Horizon Report. (2015). Higher Education Edition. Recuperado de http://www.nmc.org/ publication/nmc-horizon-report-2015-higher-education-edition/

R Core Team. (2015). R: A language and environment for statistical computing. Recuperado de https:// cran.r-project.org/doc/manuals/r-release/fullrefman.pdf

Rogmann, J. J. (2013). R- Package 'orddom': Ordinal Dominance Statistics. Recuperado de https:// cran.r-project.org/web/packages/orddom/orddom.pdf

Sein-Echaluce Lacleta, M. L., Fidalgo-Blanco, Á., García-Peñalvo, F. J., \& Conde-González, M. Á. (2016). iMOOC Platform: Adaptive MOOCs. In P. Zaphiris \& I. Ioannou (Eds.), Learning and Collaboration 
Technologies. Third International Conference, LCT 2016, Held as Part of HCl International 2016, Toronto, ON, Canada, July 17-22, 2016, Proceedings (pp. 380-390). Switzerland: Springer International Publishing. doi:https://doi.org/10.1007/978-3-319-39483-1_35

Signorell, A. (2016). R - Package 'DescTools': Tools for descriptive statistics. Recuperado de https:// cran.r-project.org/web/packages/DescTools/index.html

Stone, J. E. (2016). Awarding college credit for MOOCs: The role of the American Council on Education. Education policy analysis archives, 24, 38. doi:http://dx.doi.org/10.14507/epaa.24.1765

Teixeira, A., García-Cabot, A., García-López, E., Mota, J. \& De-Marcos, L. (2015). A new competencebased approach for personalizing moocs in a mobile collaborative and networked environment. RIED. Revista Iberoamericana de Educación a Distancia, 19(1), 143-160. doi:https://doi.org/10.5944/ ried. 19.1.14578 